\title{
Surreptitiously projecting different movies to two subsets of viewers
}

\author{
KAZUO MORI \\ Shinshu University, Nagano, Japan
}

\begin{abstract}
A new technique (manipulation of overlapping rivalrous images by polarizing filters, or MORI) has been invented for presenting on the same screen two different images that can be seen separately by two groups of viewers without their noticing the overlap. It can easily create desired artificial conflicts among viewers. Two perpendicular polarizing filters provide separate invisible channels from dual video projectors to two groups of viewers on a single screen. The basic principle of the presentation technique, details of the apparatus, and limitations are introduced. As an example of the application of this technique, an eyewitness experiment is briefly reported. The results of experiments conducted by the author and colleagues provide evidence of the effectiveness of this technique with various projectors, video materials, group sizes, and ages of participants.
\end{abstract}

In conducting psychological studies, researchers may wish to present two different stimuli to two groups of participants to create conflict between them. For example, in the field of social interaction and influence, to gauge factors such as conformity to a majority, obedience to authority, rebellion, and so forth, researchers must somehow introduce conflicts among the participants. It is more convenient for researchers to create a conflict during the experimental sessions and observe how it will be solved than to use participants who already have existing conflicting opinions with each other. Discrepancies within a group of viewers may be treated as a kind of error. If one of the viewers observes something different from what the others observed, the first viewer's observation functions practically as a misperception. At the very least, the majority of viewers tend to think that the one who observed something different must have misperceived it.

Humans are prone to mistakes, and human error has been a major research topic in psychology (see, e.g., Reason, 1990). However, it is rather difficult to observe incidental errors constantly in the laboratory setting. If two different versions of a scene can be presented secretly to the viewers, the experimenter can systematically create an artificial misperception in the laboratory. Such techniques have practical applications, especially in criminal investi-

This research was supported by Grants in Aid 08871013 and 13610081 from the Japanese Ministry of Education, Culture, Sports, Science, and Technology. I express my thanks to my students and former students in the Cognitive Psychology Laboratory, Oso-matsu Kanematsu, Detchan Mori, Misaki Yamaguchi, Hiromi J. R. Shizuyama, John Fujisawa, and Akane Yamazaki, who contributed to the invention of the technique and conducted experiments using it. I also thank Yuwen Takahashi for her helpful comments on the preparation of the English manuscript. I am also indebted to Becky Marck for her superb job in polishing up the manuscript. Correspondence concerning this article should be addressed to K. Mori, Shinshu University, Nishi-Nagano, Nagano, Japan 380-8544 (e-mail:kazmori@gipnc.shinshu-u.ac.jp). gations and/or judicial procedures involving eyewitnesses whose reports conflict with those of others due to misperception or faulty memory. Is it possible to determine which eyewitness is reporting correctly? To do so, it is necessary to have some fundamental knowledge as to what happens when two eyewitnesses observe the same event differently or report differing recollections.

Social psychologists have used confederates in their experiments to purposefully create misperception. In one of the best known experiments (Asch, 1958) concerning misperception and conformity, a group of confederates was used to show that participants tended to conform to the group consensus. In a recent example of research into children's eyewitness testimony (Goodman et al., 1998), an unfamiliar male confederate was used in a play session with child participants; the findings indicate that the older children were more accurate witnesses than the younger children, although the older children tended to produce more inaccurate information in free recall. However, using confederates in an experiment has several shortcomings. For example, participants are not so naive as to be easily fooled by them. The use of confederates also restricts experiment design. It is difficult, though not impossible, to vary interpersonal relations among "participants," or among real participants and confederates. It could be rather easy to form an unfamiliar pair between a participant and a confederate, but difficult to form a familiar pair involving a confederate. It is also very hard to find good child confederates. A better method was needed.

Various techniques for presenting two different images simultaneously have been developed in relation to 3-D display (stereoscopic) techniques. One of the most primitive is the use of two color filters, such as a red one and a green one, to view two overlapping pictures printed in green and in red. With this method, two groups of viewers wearing either green sunglasses or red sunglasses can observe two different versions printed on the same screen in red and in 
green, respectively. However, viewers can readily notice the difference between the glasses they wear, which allows them to figure out easily the difference in what they are shown. This technique has the additional shortcoming of limitations of the number of colors that can be used.

More sophisticated equipment can be devised by presenting two different images separately to two eyes to create stereoscopic vision. For example, two video images presented alternately in rapid succession can be seen separately through goggles with high-speed liquid crystal display (LCD) shutters synchronizing to only one eye. With such equipment, two full-colored video images can be presented both quasi-simultaneously and separately to two viewers sitting side by side, rather than to the two eyes of a single viewer. Since the goggles differ only in synchronization, it is impossible for the participants to tell the difference between the two types of goggles they wear. However, it is more likely that they will correctly attribute any discrepancies to the sophisticated equipment than to their "misperceptions."

A simple technique is needed to avoid making participants suspicious about tricky presentation. Since the human eye cannot detect the direction of polarized light, polarizing filters are suitable for psychological experiments. There are several advantages to using polarizing filters. Polarization does not affect color perception. In addition, two pairs of sunglasses with different polarizing directions look not only identical but also very ordinary. Participants are less likely to suspect that any presentation tricks are being played with ordinary-looking sunglasses. Therefore, using polarizing filters instead of color filters can help overcome those shortcomings stated earlier. It is also advantageous that the polarizing filters are not so expensive.

The purpose of this article is to introduce a technique that uses polarizing filters to present, on the same screen, two different images that can be viewed separately by two groups of participants without their noticing the overlap. First, the basic principle of the presentation technique is introduced. Details of the apparatus and filming methods are presented. Finally, the technique's application in an experiment (Kanematsu, Mori, \& Mori, 1996) is briefly described to provide some empirical evidence to support its usability.

\section{THE MORI TECHNIQUE}

\section{A New Presentation Procedure}

\section{Purpose of the MORI Technique}

The purpose of the MORI (manipulation of overlapping rivalrous images by polarizing filters) technique was to meet the following requirements. In order to create conflicts between two groups of viewers, it is desirable to present two different movies both simultaneously and separately to the two groups as they attend the same presentation session. The procedure must seem simple enough to prevent the participants from becoming suspicious. It is also advantageous if it can be used in a variety of psychological experiments, including those with child participants.

\section{Basic Principle}

The polarization properties of light, which are used in certain types of 3-D display, are also used in the MORI technique. Physically, light is a transverse wave that vibrates perpendicular to its direction of movement. Light is polarized in the direction in which the wave is vibrating. Usually, light comprises all polarizations. A polarizing filter allows only the light of one polarization to pass through. Therefore, once light passes through one of these filters, it cannot pass through another polarizing filter placed perpendicular to its polarization (see Figure 1). If two images are polarized in directions perpendicular to each other and are projected onto the same screen, these two images look overlapped to the naked eye. However, if a viewer wears a pair of polarizing sunglasses, only one image is seen, whereas the other is filtered out.

\section{The MORI Setup}

In the MORI technique, two different images presented on the same screen can be seen separately by two groups of viewers without their noticing that there are differing, overlapping images. Two video projectors hidden behind a half-transparent screen project polarized images that are perpendicular to each other. Each participant wears a pair of polarizing sunglasses, which look like ordinary sunglasses but can filter out one of the projected images (see Figure 2). Since polarization of light has no effect on human color or motion vision, any material can be presented in full color using the MORI technique without changing its visual characteristics.

One important point should be noted. The filtering of light by a pair of polarizing filters can be achieved as long as the filters are placed exactly perpendicular to each other. If either of the filters is tilted, the filtering will be imperfect. Filtration stays in a narrow range of $90^{\circ} \pm$ approximately $10^{\circ}$, depending on the contrast and clearness of the image. This means that if either filter is tilted more than $10^{\circ}$, the two images are seen as overlapping. Participants wearing polarizing sunglasses may occasionally tilt their heads more than $10^{\circ}$ during the presentation. Thus,

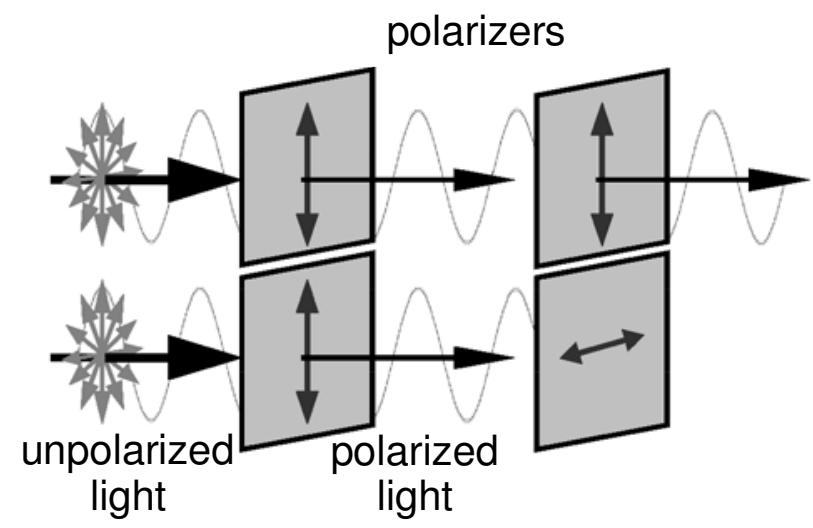

Figure 1. Polarization of light. 


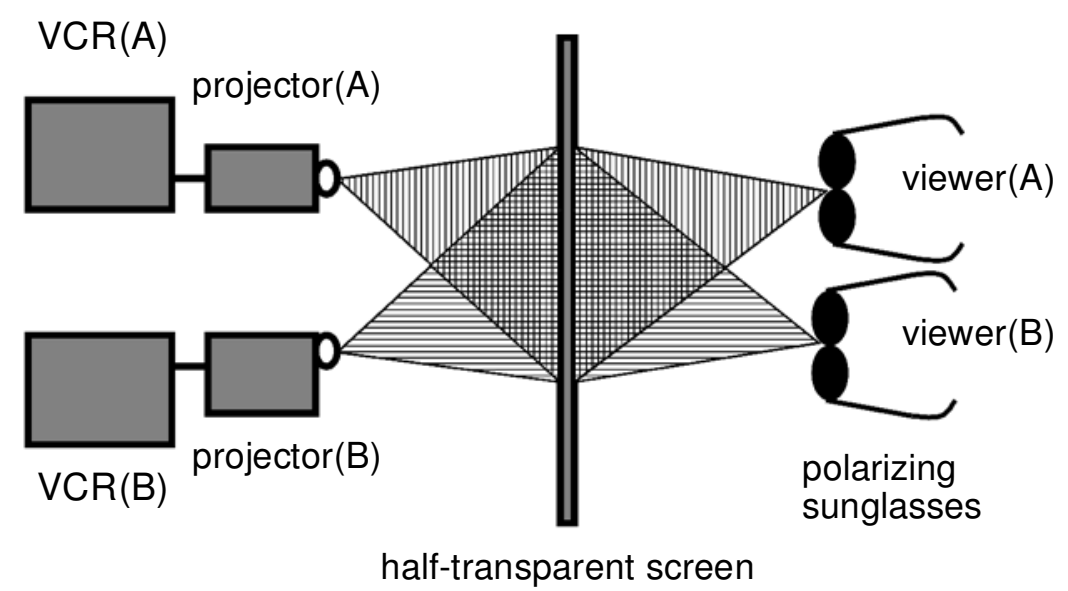

Figure 2. Overall setup of the MORI technique.

they would notice that two different images are being presented simultaneously.

There should be several remedies for this. Adjustment of the timing of the two projected movies is very important. If the two movies are synchronized perfectly, two overlapped images are unified into one on the screen during most of the presentation. Discrepancy appears only at certain points at which the experimenter has secretly inserted some variables. It is during this short period that participants might detect the duality accidentally by tilting their heads. The smaller the proportion of the discrepancy, the less chance there is that the duality will be detected. Therefore, adjustment of movie timing is important. The clearness of the images, which depends mainly on the illuminative power of the projectors, affects the possibility of the appearance of "hidden images." The brighter and clearer the projection, the greater is the chance of revealing the hidden images. Of course, there is a trade-off: If the images are not clear enough, the presentation itself is of no use. Experimenters should adjust the level of brightness according to their research objectives. A simple instruction to participants not to tilt their heads during the presentation seems effective. However, too much emphasis on not tilting the head might rouse the participants' curiosity, causing them to test the prohibited style of viewing. Instructing them to sit up straight during viewing may be more natural and unobtrusive. As will be noted in the Evaluation section, the combination of the treatments mentioned above prevented the detection of duality in actual applications. In a series of experiments with more than 300 participants conducted by the author and other researchers, no participant figured out that two different images were actually being presented.

\section{Apparatus}

Video projectors. Ordinary video projectors can be used for the MORI technique. There are at least two types of projectors on the market today: LCD projectors and DLP (digital light processing) projectors. The light beam of an LCD projector is already polarized, because it passes through a set of LCD panels within the projector. An LCD panel is composed of a matrix of small units of liquid crystals sandwiched between two polarizing filters. Therefore, an LCD panel acts as a polarizing filter.

Color video projectors contain three LCD panels, for red, green, and blue lights, respectively. In order to block all three projected beams of light with a polarizing filter, the directions of polarization produced by these three LCD panels must be identical. One of the two types of LCD projectors currently available - those in which the LCD panels are placed in different directions-is not suitable for the MORI technique. On the other hand, the type of projectors whose LCD panels are aligned is suitable for this technique. The two types of projectors can be distinguished by placing a polarizing filter in front of the projection lens and rotating it perpendicular to the projection line. If the three LCD panels are placed in the same direction, the projected image is totally blocked at a certain point during the rotation of the filter. It not, the image turns pinkish or greenish but is never completely blocked. If LCD projectors are to be used, the appropriate type should be selected.

The new DLP projector (Texas Instruments) emits an ordinary unpolarized light beam. A pair of DLP projectors can be used for the MORI technique by placing a polarizing filter in front of each projection lens, so that the pair of filters polarizes the light beams and makes them perpendicular to each other.

Half-transparent screen. The use of a half-transparent screen and projection from the rear are recommended, for the following reasons. First, ordinary screens tend to depolarize the polarized light beam on reflection, whereas half-transparent screens allow the polarized beam to pass through relatively unchanged. Second, rear projection can hide the two projectors from the viewers. A pane of plain ground glass can be used as a half-transparent screen.

The images projected onto a half-transparent screen can be seen clearly from a right angle \pm about $20^{\circ}$. It is rec- 
ommended that viewers not watch from angles wider than $30^{\circ}$ against a vertical midline of the screen. Suitable viewing distance depends on the size of the screen and the resolution of the projector as well as on the research objectives. Generally speaking, a viewing area two to five times the diagonal size of the screen is suitable.

Polarizing sunglasses. Polarizing sunglasses can be made using ordinary sunglass frames and cutouts from a sheet of polarizing filter. Plastic sheets of polarizing filter are available at a cost of about $\$ 75$ for a $1-\mathrm{mm}$-thick sheet of $30 \times 30 \mathrm{~cm}$. For sunglass frames, a 1-mm-thick sheet is recommended. Although the sheet can be easily cut with ordinary scissors, opticians do the framing job much better than laymen.

\section{Preparation of Contents}

Sideways video filming. In order to produce two perpendicularly polarized images using two LCD projectors, one of them must be set sideways. This means that the images for the projector must be filmed sideways to be projected in the upright position. Sideways video filming can be done easily by turning the video camera $90^{\circ}$ at the time of recording. Because the frame shape of an ordinary video camera is a 3:4 wider rectangle, sideways filming yields a vertically long frame. The two images are then overlaid on the same screen to produce a square-shaped intersection. Therefore, in both ordinary filming and sideways filming, targets should be framed in the center. The use of a tripod during video recording is recommended. If a ready-made movie is to be used for the presentation, digital movie editing software such as Adobe After Effects can convert ordinary angled movies into sideways ones. With DLP projectors, sideways video filming is unnecessary.

Presentation contents. Although it is possible to present two different images using the hardware mechanism of the MORI technique, the contents should be prepared carefully. It does not seem possible to make two groups of viewers believe, at postviewing conferences, that they had been shown the same material if they had actually been shown totally different movies. It is recommended that only a small number of differences be inserted within the same basic contents.

As was stated above, the blockage of the "hidden" image by polarizing sunglasses may be interrupted occasionally by the participant's accidental tilting of the head. In order to minimize the possibility that participants will notice the duality of the presentation, the two versions of video images should be edited as closely as possible in time and space. Digital video editing software, such as Adobe Premiere, is useful for the preparation of video contents.

\section{AN EXAMPLE OF AN APPLICATION}

Following Loftus's (1979) pioneering study eyewitness testimony has been a popular research topic in applied cognitive/social psychology (see, e.g., Dunning \& Stern, 1994; Gonzalez, Ellsworth, \& Pembroke, 1993; Krafka \& Penrod,
1985; Sheehan, Statham, \& Jamieson, 1991). Loftus found that memory of an event could easily be distorted by questioning following the presentation of the event. However, within the Loftus paradigm it is not possible to disentangle distortions in the memory of witnesses from those in the reporting of the event. If the experimenter can intentionally control what a witness observes irrespective of the observations of coviewers, the witness's perception, reporting, and memory in various social contexts can be investigated.

Kanematsu et al. (1996) applied the MORI technique in an eyewitness testimony experiment in which 30 pairs of undergraduates observed two movies of basically the same event with three contradictory points secretly inserted using the MORI technique. Each pair of participants was instructed to act as eyewitnesses of a criminal event that had happened in the dark. They were told to wear sunglasses while observing the videotaped event to simulate darkness. Just after the presentation, they were asked to report individually on what they had seen. Then they were allowed to discuss the event they had just observed. Fifteen of the participant pairs were instructed to come to an agreement to make a unified report, whereas the other 15 pairs were instructed to discuss what they had seen and to report again individually. The participants were invited to come to the laboratory a week later to report what they had seen the week before. At the very end of the experiment, they were asked whether they had noticed the fact that they had seen two different versions.

\section{Description of the Apparatus}

Video cameras. Two versions of a simulated criminal event were recorded using a Sony Handycam Video Hi8 (CCV-TRV91). The same type of video camera was also used to play the tapes.

Video projectors. Two LCD video projectors (JVC PD-V7) were used. Each had a 0.7-in. LCD panel with approximately 100,000 pixels. The illuminance of the projection lamp of $12 \mathrm{~V} 30 \mathrm{~W}$ was approximately $15 \mathrm{~lx}$. Both projectors were mounted on tripods and set side by side behind a half-transparent screen.

Half-transparent screen. A $20 \times 20 \mathrm{~cm}$ plain ground glass pane $5 \mathrm{~mm}$ thick was used as a half-transparent screen. It was mounted on a $180 \mathrm{~cm}$ (height) $\times 90 \mathrm{~cm}$ (width) $\times 5 \mathrm{~mm}$ (thickness) wooden panel with a $20 \times$ $20 \mathrm{~cm}$ window in the middle that served as the screen. Two versions of video images were projected onto the same half-transparent screen. Because one of the video projectors was placed sideways to make its polarized image perpendicular to that of the other, the intersection of the two images became a square on the shorter side of the two rectangles. Therefore, the screen shape was a square rather than a 3:4 rectangle.

Polarizing sunglasses. Two types of polarizing sunglasses suitable for viewing either of the video images were ordered from opticians. They were made using ordinary sunglass frames and cutouts from a sheet of polarizing filter $1 \mathrm{~mm}$ thick. 


\section{Simulated Criminal Event}

Outline of the event. In the criminal event presented on screen to the participants, a car pulled up before a pedestrian (female). The driver (male) got out of the car with a map in his hand and asked her for directions. While she was pointing out directions on the map, leaving her bag on the ground, a passenger (female) in the car quietly came out of the car, stole something from the bag, and sneaked back into the car. Then the driver bowed his thanks to the pedestrian and drove away. The pedestrian started walking again without noticing the theft.

The movie contained no audio component. The total length of the event was about $1 \mathrm{~min}$.

Three contradictory points. In order to create conflict among eyewitnesses, two versions of the same basic event were prepared and videotaped. Only the following three points differed between the two versions: (1) the color of the car (white vs. dark), (2) the clothes of the driver (a white shirt vs. a parka with stripes), and (3) the direction in which the pedestrian walked after the theft (up toward the screen vs. down away from the screen).

\section{Results}

The MORI technique worked perfectly, judging from various data obtained from the experiment. First of all, no participant reported noticing the presentation trick in postexperiment interviews. The fact that the participants did not suspect any shenanigans was also inferred from the discussion sessions; no group ended up not reaching an agreement even on the three conflicting points. If they had thought they had observed different things, they would not have reached agreement on initially divisive points. The reports given a week later also showed some evidence of their belief that they had all watched the same movie. Once they reached an agreement, those who had changed their minds tended to report the distorted memory again a week later, with or without being aware of it. Even those who changed their minds after discussion rated high on confidence in their week-later reports. These evidential behaviors were observed across the board. The results were replicated in subsequent experiments (Hirokawa, Matsuno, Mori, \& Ukita, 2003; Mori, 2003a; Mori et al., $1999)$ with varying eyewitness groupings and presentation conditions.

\section{EVALUATION OF THE MORI TECHNIQUE}

Several experiments and a demonstration have been conducted using the MORI technique; they are summarized in Table 1. The MORI technique was found to be successful in fulfilling its purpose throughout these applications, judging from the criterion of the trick's not being noticed by the participants. The results of those experiments are not the focus of this paper and, thus, are not described in detail. The technical aspects of those experiments that are more relevant to the purpose of this paper are discussed below. The results of these experiments and of a demonstration in the author's laboratory (Mori \& Komatsu, 2001) showed the applicability of the MORI technique in research involving various types of participants, apparatus, and experimenters.

The MORI technique has been used in experiments with participants of various ages and both sexes. A total of 359 undergraduates participated as eyewitnesses in groups of various sizes: in pairs (Hirokawa et al., 2003; Kanematsu et al., 1996; Mori et al., 1999; Yamazaki \& Mori, 2001), in triads (Mori, 2003a; Yamazaki \& Mori, 2001), in foursomes (Mori, 2003a), and in various sex combinations (Hirokawa et al., 2003). In an ongoing experiment (Mori, 2003b), 15 mothers and their children ranging from 6 to 12 years old participated as mother-child eyewitness pairs. In addition, 16 high school students from 12 to 17 years old attended the author's demonstration sessions of this method during a 2-day seminar in introductory psychology at Shinshu University in October 2001 (Mori \& Komatsu, 2001). They observed the video event in groups of 8 . None of them noticed the trick. These results show the wide applicability of the MORI technique across various ages and genders in comparison with other techniques used in eyewitness experiments.

This technique was also shown to be effective regardless of projector type or video contents. In one experiment (Mori et al., 1999) and in the author's demonstration (Mori \& Komatsu, 2001), a pair of much brighter LCD projectors (Yokogawa VIP4000) was used to examine the effectiveness of the MORI technique under much clearer viewing conditions and with a wider screen. The brighter the image, the greater was the chance that the duality could be detected. However, it was found that even with the

Table 1

Summary of Experiments Using the MORI Technique

\begin{tabular}{llcll}
\hline \multicolumn{1}{c}{ Source } & \multicolumn{1}{c}{ Participants } & Group Size & Projector Type & Video Contents \\
\hline Kanematsu et al. (1996) & 60 undergraduates & 2 & PD-V7 (LCD) & Hi8/analog \\
Mori (2003a) & 78 undergraduates & 3 & PD-V7 (LCD) & Hi8/analog \\
Mori (2003a) & 60 undergraduates & 4 & PD-V7 (LCD) & Hi8/analog \\
Mori et al. (1999) & 60 undergraduates & 2 & VIP4000 (LCD) & Hi8/analog \\
Hirokawa et al. (2003) & 48 undergraduates & 2 & PD-V7 (LCD) & Hi8/analog \\
Yamazaki \& Mori (2001) & 53 undergraduates & 2,3 & D-1200X (DLP) & digital animation \\
Mori (2003b) & 15 mothers/15 children & 2 & PD-V7 (LCD) & Hi8/analog \\
Mori \& Komatsu (2001) & 16 high school students & 8 & VIP4000 (LCD) & Hi8/analog \\
\hline
\end{tabular}


brighter projectors the MORI technique was effective. The simulated criminal event was made into an animated cartoon and projected by a pair of DLP projectors (Yokogawa D-1200X) in another experiment (Yamazaki \& Mori, 2001) to test the technique's reliability with different presentation equipment and materials. The results confirmed that the MORI technique can be used with brighter projectors and various visual stimuli.

Furthermore, the MORI technique can be easily employed in any laboratory setting. Hirokawa and her colleagues (Hirokawa et al., 2003) conducted an experiment at another university using the same materials as Kanematsu et al. (1996) and found that female pairs remembered better than male pairs or mixed pairs. The participants in this experiment also did not detect the presentation trick. This demonstrated the general applicability of this technique across locations and researchers.

\section{CONCLUSION}

In this article a new technique has been introduced for presenting two different visual stimuli simultaneously to two groups of viewers without their noticing the duality. The technique described is especially suitable for research requiring the creation of conflict among viewers. This technique is also advantageous in that it is easy to use in experiments with child participants. The results of several experiments conducted by the author and others provide evidence of the effectiveness of the MORI technique with various projectors, different video materials, and viewers of different ages and in different group sizes and sex combinations. It can be applied to a variety of psychological experiments in addition to the examples reported here. It is hoped that the MORI technique will expand the opportunities for reliable psychological research.

\section{REFERENCES}

AsCH, S. E. (1958). Effects of group pressure upon modification and distortion of judgments. In E. E. Maccoby, T. M. Newcomb, \& E. L. Hartley (Eds.), Readings in social psychology (3rd ed., pp. 174-183). New York: Holt, Rinehart \& Winston.

DunNING, D., \& STERN, L. B. (1994). Distinguishing accurate from inaccurate eyewitness identifications via inquiries about decision processes. Journal of Personality \& Social Psychology, 67, 818-835.
Gonzalez, R., Ellsworth, P. C., \& Pembroke, M. (1993). Response biases in lineups and showups. Journal of Personality \& Social Psychology, 64, 525-537.

Goodman, G. S., Tobey, A. E., Batterman-Faunce, J. M., Orcutt, H., Thomas, S., Shapiro, C., \& SAChSEnMAIER, T. (1998). Face-to-face confrontation: Effects of closed-circuit technology on children's eyewitness testimony and jurors' decisions. Law \& Human Behavior, 22, 165-203.

Hirokawa, K., Matsuno, E., Mori, K., \& UKitA, J. (2003). Effects of masculinity and femininity on recalled memories in experimental collaborative eyewitness testimony. Manuscript submitted for publication. Kanematsu, H., Mori, K., \& Mori, H. (1996). Kotonaru jitai wo mokugekishita futari no mokugekisha no hanashiai niyoru kioku no hen-you [Memory distortion in eyewitness pairs who observed nonconforming events and discussed them]. Cognitive Studies: Bulletin of the Japanese Cognitive Science Society, 3, 29-40. [English version available in Journal of Faculty of Education (2003, Shinshu University), 109, 75-84]

KrafkA, C., \& Penrod, S. (1985). Reinstatement of context in a field experiment on eyewitness identification. Journal of Personality \& Social Psychology, 49, 58-69.

LofTus, E. F. (1979). Eyewitness testimony. Cambridge, MA: Wiley

MORI, K. (2003a). Memory distortion of isolated eyewitnesses in an experimental collaborative eyewitness testimony. Manuscript submitted for publication.

Mori, K. (2003b). "No, Mum. It was a white car”: What happens if mother and child dyads witnessed the same event differently? Proceeding s of the Fourth Tsukuba International Conference on Memory, Tsukuba, Japan. Available at http://www.human.tsukuba.ac.jp/tic4/ html/10.html.

Mori, K., Kanematsu, H., Mori, H., Yamaguchi, M., Shizuyama, H., \& FUJISAWA, T. (1999). Memory distortion in eyewitnesses who observed non-conforming events experimentally produced by polarizing filters. Proceedings of the Second International Conference on Cognitive Science and the Sixteenth Annual Meeting of the Japanese Cognitive Science Society Joint Conference (pp. 219-222). Conference held at Waseda University, Tokyo.

MORI, K., \& KOMATSU, S. (2001). [An introductory seminar on experimental studies in human memory for high school students.] Unpublished demonstration.

ReAson, J. (1990). Human error. Cambridge: Cambridge University Press.

Sheehan, P. W., Statham, D., \& Jamieson, G. A. (1991). Pseudomemory effects and their relationship to level of susceptibility to hypnosis and state instruction. Journal of Personality \& Social Psychology, 60, 130-137.

YAMAZAKI, A., \& MORI, K. (2001). Memory distortion in eyewitness pairs and triads who observed nonconforming events presented in animated movies. Unpublished manuscript.

(Manuscript received April 1, 2002; revision accepted for publication December 20, 2002.) 